\title{
Simulación del proceso de producción de anhídrido maleico mediante el simulador ChemCAD $^{\circ}$
}

\author{
Simulation of the Maleic Anhydride Production \\ Process through ChemCAD Simulator
}

\section{Amaury Pérez Sánchez ${ }^{\mathrm{ac}}$, Elizabeth Ranero González ${ }^{\text {ad, }}$ Eddy Javier Pérez Sánchez ${ }^{\text {be }}$}

\footnotetext{
a Departamento de Ingeniería Química, Facultad de Ciencias Aplicadas, Universidad de Camagüey “Ignacio Agramonte Loynaz”, Camagüey, Cuba ${ }^{b}$ Empresa Servicios Automotores S.A., Ciego de Ávila, Cuba

camaury.psanchez@reduc.edu.cu | https://orcid.org/0000-0002-0819-6760

${ }^{\mathrm{d}}$ https://orcid.org/0000-0001-9755-0276

e https://orcid.org/0000-0003-4481-1262
}

\section{RESUMEN}

El anhídrido maleico es una un producto químico con un amplio rango de aplicaciones como compuesto intermedio en la producción de resinas, polímeros, barnices y pinturas. En el siguiente trabajo se llevó a cabo la simulación del proceso de producción del anhídrido maleico a partir de la oxidación catalítica de benceno mediante el simulador ChemCAD ${ }^{\circledR}$, versión 5.2.0, con

Citation: Pérez-Sánchez, A., Ranero-González, E. y Pérez-Sánchez, E. D. (2021). Simulación del proceso de producción de anhídrido maleico mediante el simulador ChemCAD:. Mutis, 11(2), 8-21. https://doi. org/10.21789/22561498.1752

Recibido: 25 de agosto de 2020 Aceptado: 25 de marzo de 2021

Copyright: @2021. Pérez-Sánchez, A., Ranero-González, E. y PérezSánchez, E. D. (2021). This is an open-access article, which permits unrestricted use, distributions and reproduction in any medium, provided the original author and source are credited.

Competing Interests: The authors have no conflict of interest. el fin de realizar los balances de masa de todas las corrientes de proceso, determinar el consumo de los servicios auxiliares en los intercambiadores de calor y conocer los parámetros de diseño más importantes del equipamiento empleado. Se llevaron a cabo dos estudios de sensibilidad: influencia de la temperatura y presión de operación del absorbedor sobre la cantidad a obtener de anhídrido maleico por la corriente del fondo de este equipo. Se obtienen $5.027,14 \mathrm{~kg} / \mathrm{h}$ de anhídrido maleico por la corriente del tope de la columna de destilación con una pureza del 97,45 \%. El absorbedor debe operar a una 
temperatura cercana a 20 ㄷ y una presión de 5 bar para reducir las pérdidas de anhídrido maleico e incrementar las ganancias económicas del proceso.

Palabras claves: anhídrido maleico, benceno, $C$ emCAD ${ }^{\circledR}$, estudio de sensibilidad, simulación.

\section{ABSTRACT}

Maleic anhydride is a chemical product with a wide range of applications in the production of resins, polymers, varnishes, and paints. This work presents a simulation of the maleic anhydride production process from the catalytic oxidation of benzene carried out in ChemCAD ${ }^{\circledR}$ simulator, version 5.2.0, in order to perform the mass balances of all the process streams, determine the consumption of utilities in the heat exchangers, and establish the most important design parameters of the equipment used. Two sensitivity studies were carried out: influence of the operating temperature and pressure of the absorber on the amount of maleic anhydride to be obtained in the bottom stream of this equipment. Around $5,027.14 \mathrm{~kg} / \mathrm{h}$ of maleic anhydride were obtained on the top stream of the distillation column, with a purity of $97.45 \%$. Based on our findings, the absorber must operate at a temperature near $20 \stackrel{\circ}{ } \mathrm{C}$ and a pressure of 5 bar to reduce losses of maleic anhydride and increase the economic profits of the production process.

Keywords: Maleic anhydride, benzene, $C$ hemCAD ${ }^{\circledR}$, sensitivity study, simulation.

\section{INTRODUCCIÓN}

La oxidación de compuestos orgánicos como el benceno en fase de vapor es una reacción valiosa desde el punto de vista industrial debido a que los productos obtenidos, tales como el anhídrido maleico (AM) y el anhídrido ftálico, son intermediarios importantes (Uraz \& Atalay, 2007).

El $\mathrm{AM}$ es un compuesto químico empleado para producir resinas, recubrimientos superficiales, aditivos de lubricantes y de alimentos, pinturas, barnices y sustancias químicas para la agricultura (Speight, 2002). Este se obtiene industrialmente mediante la oxidación de hidrocarburos específicos en fase gaseosa. El benceno resultó ser la materia prima predominante para la obtención de AM (Uraz \& Atalay, 2007, 2012), aunque posteriormente la oxidación catalítica de hidrocarburos C-4 (fundamentalmente butano) ganó en importancia (Fernández et al., 2010; Pugsley et al., 1992; Tandioy et al., 2009).

La tecnología del reactor empleado en el proceso de producción del anhídrido maleico involucra tanto las configuraciones de lecho fijo como fluidizado, aunque también se ha desarrollado la tecnología de lecho fluidizado circulante. Para la oxidación selectiva del benceno, la tecnología del proceso involucra la configuración de lecho fijo, donde la reacción ocurre en fase gaseosa a 400-450 ㄷ en un reactor de flujo en pistón multitubular. El catalizador, esencialmente un óxido mixto de vanadio/molibdeno (V/Mo) es depositado en un soporte inerte y altamente conductivo para permitir una mejor remoción del calor, el cual proviene de la reacción selectiva y también de las reacciones indeseables (combustión total del benceno y los productos). Bajo estas condiciones, la conversión del benceno es casi total (cerca de $96 \%$ ) y la selectividad del AM puede alcanzar el $73 \%$ (Nosakhare et al., 2017).

La propagación del uso de los computadores y ordenadores digitales ha impulsado el desarrollo de una gran variedad de herramientas para la solución de problemas de ingeniería y aquellos propios de las etapas iniciales del desarrollo de procesos químicos. En los últimos años, las técnicas de simulación se han afianzado lo suficiente, provocando que su uso se haya extendido a diversos campos de la ciencia y la técnica. Básicamente, la simulación es el estudio de un sistema a través de un modelo, el cual, en cualquier dominio de la ciencia, es el resultado logrado por el especialista al establecer las complejas conexiones entre un objeto de estudio del mundo real y una representación conceptual de ese objeto, esto es, el modelo (Rabelo, 2016). 
En la actualidad, el empleo de la simulación de procesos químicos ha ganado en importancia gracias a que se trata de una actividad confiable, económica y que demanda menor tiempo para la obtención del modelo representativo del sistema bajo estudio. La simulación facilita el estudio de la influencia de diferentes parámetros de entrada sobre cualquier proceso químico, ayudando a comprender el rango de variables a partir del cual el proceso funciona adecuadamente, entregando así los mejores resultados en términos de cantidad y calidad (Greeshma et al., 2014).

Entre los simuladores más utilizados en la actualidad se encuentra el ChemCAD ${ }^{\circ}$, el cual ha sido empleado para simular diferentes procesos productivos, entre los que se encuentran la fabricación de acetaldehído (Eliasson, 2010), biodiésel (Chilev \& Simeonov, 2014), ácido monocloroacético (Greeshma et al., 2014), epiclorohidrina (Almena \& Martín, 2016), estireno (Pérez et al., 2017) y ácido acrílico (Pérez et al., 2019), así como la deshidratación de propanol (Wyczesany, 2017).

En el presente trabajo se lleva a cabo la simulación del proceso de producción de AM a partir de la oxidación parcial catalítica del benceno, empleando para ello el simulador de procesos ChemCAD $^{\circ}$ versión 5.2.0, con el objetivo de conocer la composición másica de cada una de las corrientes intermedias y finales del proceso de producción, determinar los principales parámetros de diseño de los equipos involucrados en el proceso y conocer el caudal másico de los servicios auxiliares consumidos en los intercambiadores de calor. Se efectuaron además dos estudios de sensibilidad en los cuales se evaluará la influencia del incremento tanto de la temperatura como de la presión de operación del absorbedor sobre la cantidad de AM a obtener por la corriente del fondo de este equipo.

\section{MATERIALES Y MÉTODOS}

\section{Propiedades del anhídrido maleico}

El AM se presenta en forma de hojuelas cristalinas incoloras o blancas con un olor acre (pungente). Este es un compuesto químico tóxico e irritante a la piel y las membranas mucosas, que conduce a la ocurrencia de quemaduras y ulceraciones. La tabla 1 muestra las propiedades químico-físicas más importantes del AM.

Tabla 1. Propiedades químico-físicas más importantes del AM

\begin{tabular}{|c|c|}
\hline Propiedad & Valor \\
\hline Fórmula molecular & $\mathrm{C}_{4} \mathrm{H}_{2} \mathrm{O}_{3}$ \\
\hline Masa molar & $98,06 \mathrm{~g} / \mathrm{mol}$ \\
\hline Apariencia & Cristales blancos \\
\hline Densidad & $1,48 \mathrm{~kg} / \mathrm{L}$ \\
\hline Punto de fusión & $52,85 \stackrel{\circ}{ } \mathrm{C}$ \\
\hline Punto de ebullición & 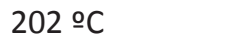 \\
\hline Calor específico (sólido) & $0,285 \mathrm{kcal} / \mathrm{kg} \stackrel{\mathrm{C}}{ }$ \\
\hline Calor específico (líquido) & $0,396 \mathrm{kcal} / \mathrm{kg} \stackrel{\circ}{ } \mathrm{C}$ \\
\hline Calor de formación (a partir de benceno) & $-1.875,0 \mathrm{~kJ} / \mathrm{mol}$ \\
\hline Calor de combustión & $-1.390,0 \mathrm{~kJ} / \mathrm{mol}$ \\
\hline Calor de vaporización & $54,8 \mathrm{~kJ} / \mathrm{mol}$ \\
\hline Calor de fusión & $13,66 \mathrm{KJ} / \mathrm{mol}$ \\
\hline Calor de hidrólisis & $34,9 \mathrm{~kJ} / \mathrm{mol}$ \\
\hline
\end{tabular}

Fuente: elaboración propia con base en Kirk et al. (2004) y Nosakhare et al. (2017)

\section{Descripción del proceso de producción del anhídrido maleico a partir de benceno}

La figura 1 muestra un diagrama de flujo con las principales etapas involucradas en el proceso de producción de AM. Para la elaboración y el diseño del proceso de producción que a continuación se describe, se empleó información publicada por Nosakhare et al. (2017) y Turton et al. (2018). 
Figura 1. Diagrama de flujo del proceso de producción de anhídrido maleico a partir de benceno

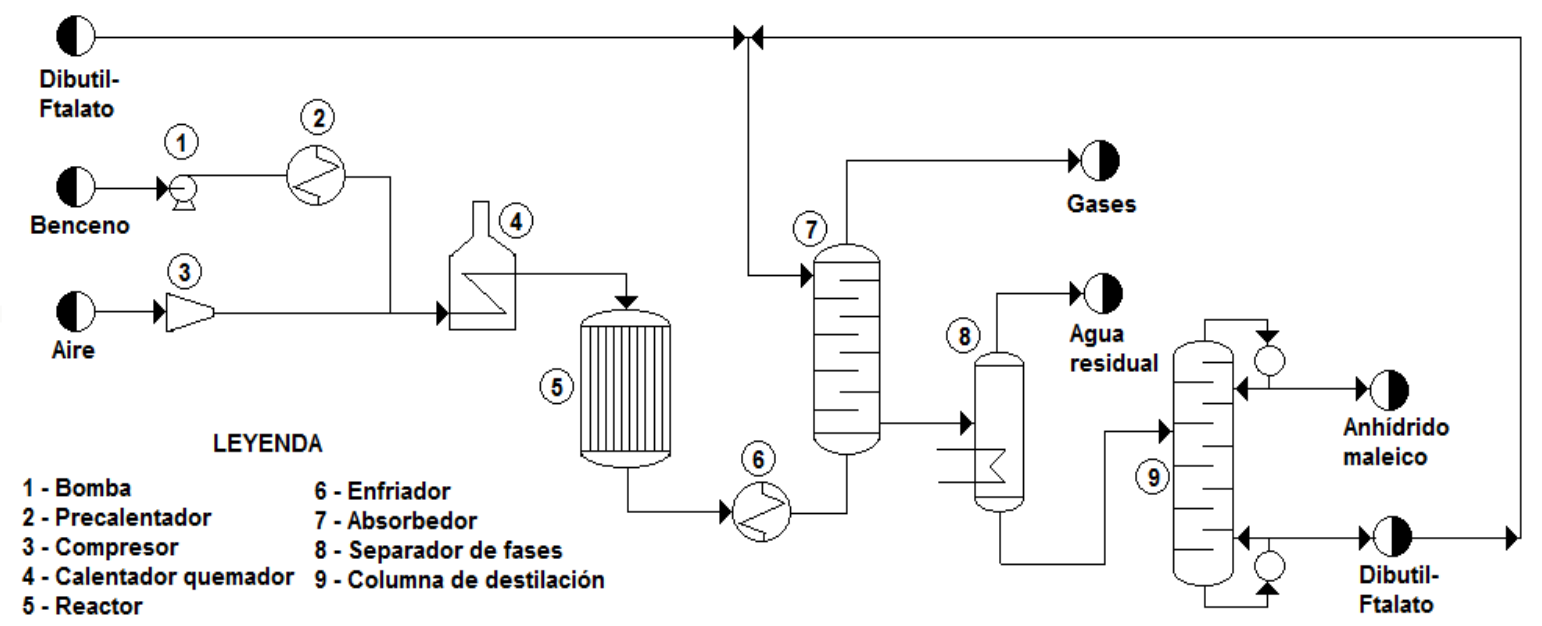

Fuente: elaboración propia.

En primer lugar, se bombean $4.435,87 \mathrm{~kg} / \mathrm{h}$ de benceno hacia un intercambiador de calor de tubo y coraza (precalentador) por medio de una bomba centrífuga, donde ocurre su calentamiento hasta 120 C empleando vapor de agua saturado a $135 \stackrel{\circ}{\circ}$ como agente de calentamiento. Por otro lado, se comprime aire seco atmosférico hasta 3 bar de presión por medio de un compresor centrífugo. Posteriormente, la corriente de benceno precalentada es mezclada con la corriente de aire comprimido con una relación másica aire/ benceno de 18,2:1. La mezcla resultante benceno/ aire es enviada luego hacia un calentador quemador, donde se calienta hasta 371 으, ocurriendo la vaporización total del benceno. El benceno vaporizado y el aire seco caliente son enviados a continuación hacia un reactor catalítico de lecho empacado, donde reaccionan en presencia de un catalizador colocado en tubos de $25 \mathrm{~mm}$ de diámetro y 3,2 m de largo, formando una mezcla gaseosa que contiene fundamentalmente AM, agua $\left(\mathrm{H}_{2} \mathrm{O}\right)$ y dióxido de carbono $\left(\mathrm{CO}_{2}\right)$.

La temperatura de reacción promedio es de 380 ㄷ C y esta debe ser controlada en el rango de 350-400 oC. Debido a que la reacción es de tipo exotérmica, se hace circular sal fundida (una mezcla de nitrito de sodio y nitrato de sodio) por la chaqueta del reactor para mantener la temperatura de reacción en el valor establecido. En el reactor se obtiene una tasa de conversión benceno/anhídrido maleico de 93,2\%. Los gases de reacción obtenidos a la salida del reactor, que contienen además oxígeno $\left(\mathrm{O}_{2}\right)$, nitrógeno $\left(\mathrm{N}_{2}\right)$, benceno y quinona, provenientes de las reacciones secundarias, abandonan el reactor y son enfriados hasta 54 으 en un intercambiador de calor de tubo y coraza (enfriador) que emplea agua de enfriamiento a 30 ㄷ, recuperándose en estado líquido el $100 \%$ del AM alimentado.

El efluente obtenido a la salida del enfriador es enviado hacia una columna de absorción (absorbedor) donde establece contacto con el solvente dibutil-ftalato (DF), recuperándose de esta manera alrededor de 99,4 \% del AM administrado. En el absorbedor se emplean $800 \mathrm{~kg} / \mathrm{h}$ del solvente DF fresco, el cual se une al reciclo que se obtiene por el fondo de la columna de destilación, que contiene DF con un 99,9\% de pureza. Este equipo opera a una temperatura y presión promedios de 42 으 y 4 bar, respectivamente. La corriente que sale por el tope del absorbedor, rica en gases incondensables $\left(\mathrm{CO}_{2}, \mathrm{O}_{2}\right.$ y $\left.\mathrm{N}_{2}\right)$, es venteada hacia la atmósfera, por lo que se recomienda efectuar un estudio posterior que involucre la implementación de operaciones unitarias para purificar y recuperar el $\mathrm{CO}_{2}$ que sale por esta corriente. De otro lado, la corriente que sale por el fondo del absorbedor, que contiene fundamentalmente AM, DF y agua, es enviada hacia un separador de fases para eliminar hasta un $95 \%$ del agua que se alimenta, así como también la totalidad del benceno y cerca de $85 \%$ de la quinona. La mezcla obtenida por el fondo del separador de fases se 
enviada hacia una columna de destilación de platos, la cual opera a 1 atm de presión, mientras que la corriente del tope es enviada hacia la planta de tratamiento de residuales.

Alrededor del 99,9\% del DF alimentado a la columna de destilación es recuperado por la corriente de fondo de este equipo. Este DF recuperado recircula de nuevo al absorbedor con una pureza cercana al 99,9\%. El producto del tope de la columna de destilación, que se encuentra en estado líquido, contiene AM con 97,5 $\%$ de pureza, siendo el agua y la quinona las principales impurezas encontradas.

\section{Catalizador}

El catalizador empleado contiene entre 70 y $75 \%$ de pentóxido de vanadio $\left(\mathrm{V}_{2} \mathrm{O}_{5}\right)$ y entre 25 y $30 \%$ de trióxido de molibdeno $\left(\mathrm{MoO}_{3}\right)$ colocado sobre un soporte inerte. El diámetro de los gránulos es de $5 \mathrm{~mm}$ y la temperatura máxima a la cual puede estar expuesto el catalizador sin que ocurra daño irreversible (sinterización) es de 650 ㄷ․ La tabla 2 muestra las propiedades físicas del catalizador empleado.
Tabla 2. Propiedades físicas del catalizador utilizado

\begin{tabular}{lc}
\hline Propiedad & Valor \\
\hline Esfericidad de la partícula (adimensional) & 0,80 \\
Densidad del sólido $(\mathrm{kg} / \mathrm{m} 3)$ & 2.500 \\
Densidad a granel $(\mathrm{kg} / \mathrm{m} 3)$ & 1.250 \\
Capacidad calórica del sólido (kJ/kg o C) & 250 \\
Fracción hueca del lecho (adimensional) & 0,50 \\
\hline
\end{tabular}

Fuente: elaboración propia con base en Nosakhare et al. (2017).

\section{Reacciones químicas y velocidad de reacción}

La tabla 3 muestra las diferentes reacciones químicas que ocurren en el reactor, así como la expresión del coeficiente cinético de la reacción (Turton et al., 2018), la extensión considerada y el calor de reacción (Kirk et al., 2004).

Tabla 3. Reacciones químicas que ocurren en el reactor, con su cinética de reacción y extensión

\begin{tabular}{|c|c|c|c|}
\hline Reacción y cinética & Ec. & Extensión & $\begin{array}{c}\text { Calor de reacción } \\
(\mathrm{kJ} / \mathrm{mol})\end{array}$ \\
\hline \multicolumn{4}{|l|}{ Formación de AM } \\
\hline $\begin{array}{l}\mathrm{C}_{6} \mathrm{H}_{6}(\mathrm{~g})+4,5 \mathrm{O}_{2}(g) \stackrel{k_{1}}{\longrightarrow} \mathrm{C}_{4} \mathrm{H}_{2} \mathrm{O}_{3}(g)+2 \mathrm{CO}_{2}(g)+2 \mathrm{H}_{2} \mathrm{O}(\mathrm{g}) \\
k_{1}=7,7 \times 10^{6} \mathrm{exp}(-25143 / R T)\end{array}$ & (1) & $93,20 \%$ & $-1875,0$ \\
\hline \multicolumn{4}{|l|}{ Combustión del benceno } \\
\hline $\begin{array}{l}C_{6} H_{6}(g)+7,5 O_{2}(g) \stackrel{k_{2}}{\longrightarrow} 6 \mathrm{CO}_{2}(g)+3 \mathrm{H}_{2} O(g) \\
k_{2}=6,31 \times 10^{7} \exp (-29850 / R T)\end{array}$ & $(2)$ & $3,35 \%$ & $-3267,6$ \\
\hline \multicolumn{4}{|l|}{ Combustión del AM } \\
\hline $\begin{array}{l}\mathrm{C}_{4} \mathrm{H}_{2} \mathrm{O}_{3}(g)+3 \mathrm{O}_{2}(g) \stackrel{k_{3}}{\longrightarrow} 4 \mathrm{CO}_{2}(g)+\mathrm{H}_{2} \mathrm{O}(\mathrm{g}) \\
k_{3}=2,33 \times 10^{4} \exp (-21429 / R T)\end{array}$ & (3) & $30,00 \%$ & $-1390,0$ \\
\hline \multicolumn{4}{|l|}{ Formación de quinona } \\
\hline $\begin{array}{l}C_{6} H_{6}(g)+1,5 O_{2}(g) \stackrel{k_{4}}{\longrightarrow} C_{6} H_{4} O_{2}(g)+H_{2} O(g) \\
k_{4}=7,20 \times 10^{5} \exp (-27149 / R T)\end{array}$ & (4) & $1,66 \%$ & $-122,9$ \\
\hline
\end{tabular}


Las unidades de la velocidad de reacción $\left(r_{i}\right)$ son $\mathrm{kmol} /$ $\mathrm{m}^{3}$ (reactor)·s, la energía de activación está dada en $\mathrm{kcal} / \mathrm{kmol}$, las unidades de $\mathrm{k}_{\mathrm{i}}$ son $\mathrm{m}^{3}$ (gas) $/ \mathrm{m}^{3}$ (reactor). $\mathrm{s}$ y las unidades de concentración corresponden a $\mathrm{kmol} / \mathrm{m}^{3}$ (gas).

\section{Equipamiento principal utilizado en el proceso de producción y sus funciones}

\section{Bomba centrífuga}

Se emplea para incrementar la presión del benceno líquido desde la presión atmosférica hasta la presión de reacción (3 bar), para así tomar en cuenta la caída de presión dentro del recipiente de reacción. Si la presión de alimentación del benceno al reactor se encuentra por debajo de la presión atmosférica, la caída de presión dentro del reactor puede ocasionar contracción y conducir a eventos catastróficos. Este equipo presenta una eficiencia de $65 \%$ (Turton et al., 2018).

\section{Compresor}

Se usa para incrementar la presión del aire de alimentación hasta la presión del reactor especificada (3 bar). El equipo empleado es un compresor centrífugo con una eficiencia adiabática de $80 \%$.

\section{Precalentador}

Se emplea para precalentar la corriente de benceno desde 30 hasta $120 \stackrel{\circ}{ }$, utilizando vapor de agua como agente de calentamiento. Es un intercambiador de calor de tubo y coraza del tipo TEMA BEM con un área total de intercambio de calor de $130 \mathrm{~m}^{2}$ (TEMA, 1999). Se consideró un coeficiente global de transferencia de calor de $750 \mathrm{~W} / \mathrm{m}^{2} \cdot \mathrm{K}(\mathrm{Cao}, 2010)$.

\section{Calentador quemador}

La mezcla benceno/aire es calentada hasta 371 ㄷ $\mathrm{C}$ por medio de un calentador quemador, el cual consume gas natural como combustible para efectuar el calentamiento. El equipo empleado registra una eficiencia de $80 \%$.

\section{Reactor}

En este equipo se llevan a cabo las reacciones (1)-(4). Se escoge una configuración del reactor de lecho empacado del tipo isotérmico.

\section{Enfriador}

Se emplea para enfriar la mezcla gaseosa de salida del

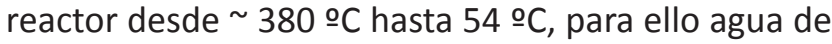

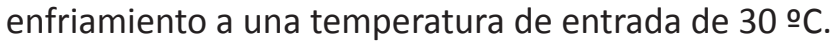
El simulador ChemCAD ${ }^{\circledR}$ calculará la cantidad de agua de enfriamiento necesaria para cumplir con este servicio. La temperatura de salida del agua de enfriamiento en el enfriador no deberá superar 50 ㅇ․ Es un intercambiador de calor de tubo y coraza del tipo TEMA AES con un área total de intercambio de calor de $280 \mathrm{~m}^{2}$ (TEMA, 1999). Se consideró un coeficiente global de transferencia de calor de $400 \mathrm{~W} / \mathrm{m}^{2} \cdot \mathrm{K}$ (Cao, 2010).

\section{Absorbedor}

Este equipo es una columna de platos perforados que opera a 4 bar y 42 으, y es donde se lleva a cabo la absorción del AM alimentado empleando DF como solvente. La corriente rica en AM es alimentada por el fondo, mientras que la corriente de DF entra por el tope del equipo. El simulador ChemCAD ${ }^{\circledR}$ determinará la cantidad total de etapas o platos necesaria para llevar a cabo la operación de absorción.

\section{Separador de fases}

Se utiliza para separar fundamentalmente el componente agua de los demás compuestos químicos presentes en la corriente de salida del absorbedor.

\section{Columna de destilación}

Es una columna de platos que opera a 1 atm de presión, y es donde ocurre la separación final del AM del solvente DF. El simulador ChemCAD ${ }^{\circledR}$ determinará la cantidad de platos o etapas que necesita este equipo para cumplir con la demanda del proceso de destilación.

\section{Selección del modelo termodinámico}

Se escogió el modelo termodinámico UNIFAC con asociación en fase de vapor para llevar a cabo la simulación en el simulador ChemCAD ${ }^{\circledR}$. Este modelo se ajusta adecuadamente a los intervalos de presión y temperatura manejados en el proceso, así como también a los tipos de sustancias utilizados, según recomendación realizada por el propio simulador a partir del empleo de la opción "K-Value Wizard" contenida en el software. 


\section{Estudio de sensibilidad}

Se efectuaron dos estudios de sensibilidad utilizando la opción "Sensitivity Study" contenida en el simulador ChemCAD ${ }^{\circledR}$. En el primero de ellos se evaluó la influencia que presenta un incremento de la temperatura de operación de la columna de absorción (absorbedor) sobre la cantidad de AM a obtener por la corriente del fondo de este equipo, a partir de la aplicación de un rango de temperatura que osciló entre 30-100 ㄷ y manteniendo constante la presión de este equipo en un valor de 4 bar. En el segundo estudio se valoró la influencia que presenta un incremento de la presión de operación del absorbedor sobre el flujo másico a obtener de AM por la corriente del fondo, mediante la variación de este parámetro entre 1-10 bar y manteniendo constante la temperatura de operación en 42 ․ C. Además, se determinó el impacto económico de ambos estudios de sensibilidad.

\section{RESULTADOS Y DISCUSIÓN}

A continuación, se muestran los principales resultados obtenidos durante la simulación del proceso de producción de AM con respecto a los balances de masa en cada una de las corrientes principales, los parámetros de rendimiento de los equipos, las curvas de calor de los intercambiadores de calor y los estudios de sensibilidad efectuados. La figura 2 muestra el diagrama de flujo del proceso de producción obtenido mediante el simulador ChemCAD ${ }^{\circledR}$.

Figura 2. Diagrama de flujo del proceso de producción de $A M$ a partir de benceno obtenido en el simulador $C h e m C A D^{\circledR}$

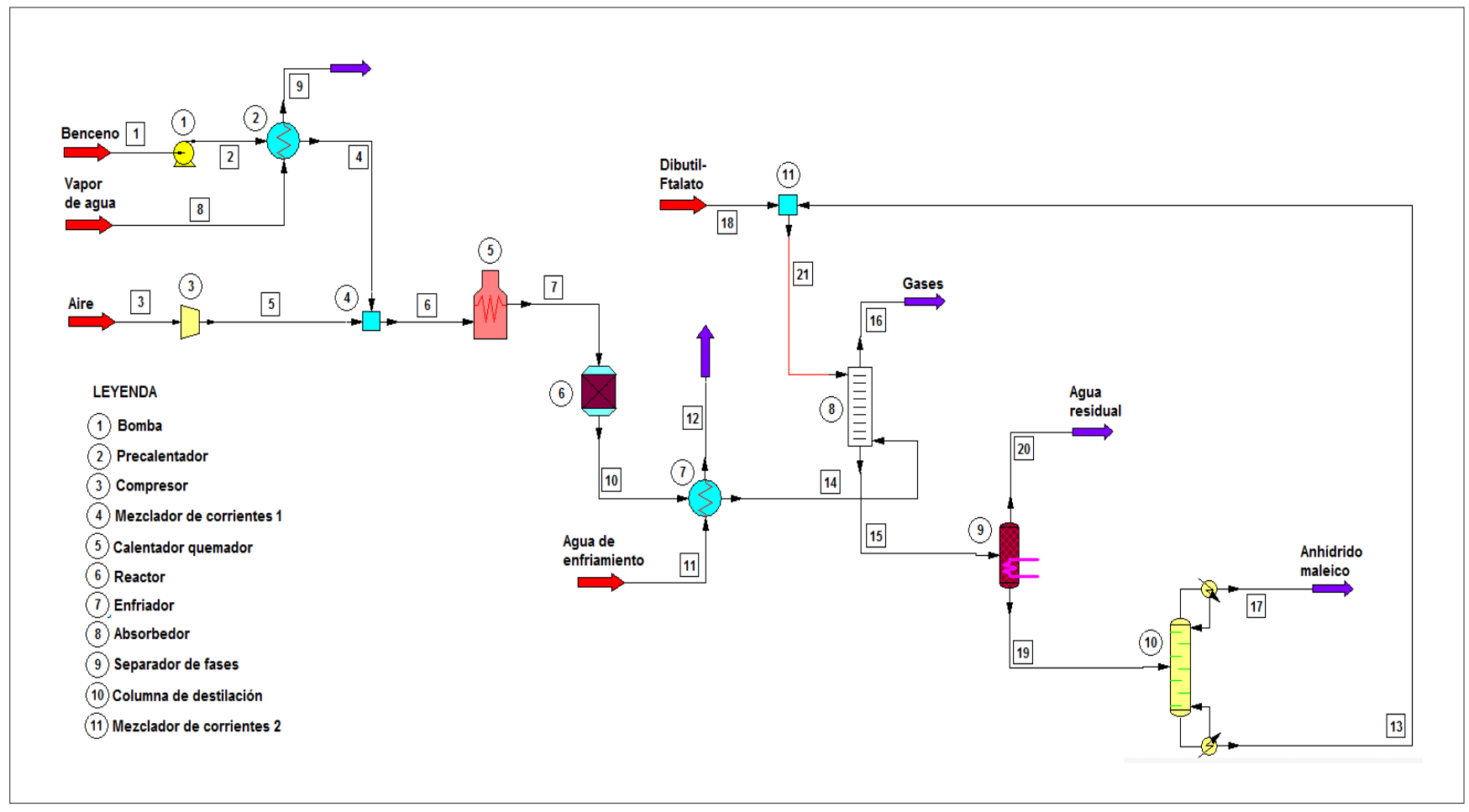

Fuente: elaboración propia.

\section{Corrientes de proceso}

La tabla 4 expone la cantidad manejada de cada compuesto químico, así como la temperatura, presión y fracción de vapor de cada corriente de proceso. 
Tabla 4. Resultados obtenidos de las principales corrientes de proceso

\begin{tabular}{|c|c|c|c|c|c|c|}
\hline \multirow{2}{*}{ Variable } & \multicolumn{6}{|c|}{ Número de la corriente (ver figura 2) } \\
\hline & 1 & 2 & 3 & 4 & 5 & 6 \\
\hline Temperatura (으) & 30 & 30,16 & 30 & 120 & 170 & 169,21 \\
\hline Presión (bar) & 1 & 3 & 1 & 2,8 & 3 & 3 \\
\hline Fracción de vapor & 0 & 0 & 1 & 1 & 1 & 1 \\
\hline Compuesto & \multicolumn{6}{|c|}{ Caudal (kg/h) } \\
\hline Benceno & 4.436 & 4.436 & - & 4.436 & - & 4.436 \\
\hline DF & - & - & - & - & - & - \\
\hline Dióxido de carbono & - & - & - & - & - & - \\
\hline Agua & - & - & - & - & - & - \\
\hline AM & - & - & - & - & - & - \\
\hline Oxígeno & - & - & $16.963,97$ & - & $16.963,97$ & $16.963,97$ \\
\hline Quinona & - & - & - & - & - & - \\
\hline Nitrógeno & - & - & $63.816,83$ & - & $63.816,83$ & $63.816,83$ \\
\hline Total & 4.436 & 4.436 & $80.780,80$ & 4.436 & $80.780,80$ & $85.216,80$ \\
\hline \multirow{2}{*}{ Variable } & \multicolumn{6}{|c|}{ Número de la corriente (ver figura 2) } \\
\hline & 7 & 10 & 14 & 21 & 15 & 16 \\
\hline Temperatura (으) & 380 & 380 & 54 & 320,30 & 42 & 42 \\
\hline Presión (bar) & 3 & 2,8 & 2,8 & 5 & 4 & 4 \\
\hline Fracción de vapor & 1 & 1 & 0,32 & 0 & 0 & 1 \\
\hline Compuesto & \multicolumn{6}{|c|}{ Caudal (kg/h) } \\
\hline Benceno & 4.436 & 79,40 & 79,40 & - & 18,89 & 60,51 \\
\hline DF & - & - & - & $7.907,50$ & $7.905,14$ & 2,36 \\
\hline Dióxido de carbono & - & 5.161 & 5.161 & - & 0,24 & $5.160,76$ \\
\hline Agua & - & $2.026,76$ & $2.026,76$ & - & $1.415,99$ & 610,77 \\
\hline AM & - & $5.189,93$ & $5.189,93$ & 4,90 & $5.161,91$ & 32,92 \\
\hline Oxígeno & $16.963,97$ & $8.840,98$ & $8.840,98$ & - & 0,05 & $8.840,93$ \\
\hline Quinona & - & 101,90 & 101,90 & 0,01 & 100,64 & 1,27 \\
\hline Nitrógeno & $63.816,83$ & $63.816,83$ & $63.816,83$ & - & 0,11 & $63.816,72$ \\
\hline Total & $85.216,80$ & $85.216,80$ & $85.216,80$ & $7.912,41$ & $14.602,97$ & $78.526,24$ \\
\hline \multirow{2}{*}{ Variable } & \multicolumn{6}{|c|}{ Número de la corriente (referirse a la Figura 2) } \\
\hline & 19 & 20 & 17 & 13 & 18 & \\
\hline Temperatura $(\stackrel{\circ}{\mathrm{C}})$ & 60 & 70 & 204,21 & 342,32 & 30 & \\
\hline Presión (bar) & 1 & 1 & 1 & 1,1 & 4 & \\
\hline Fracción de vapor & 0 & 0 & 0 & 0 & 0 & \\
\hline Compuesto & \multicolumn{6}{|c|}{ Caudal (kg/h) } \\
\hline Benceno & - & 18,89 & - & - & - & \\
\hline DF & $7.114,62$ & 790,51 & 7,11 & $7.107,51$ & 800 & \\
\hline Dióxido de carbono & - & 0,24 & - & - & - & \\
\hline Agua & 70,80 & $1.345,20$ & 70,80 & - & - & \\
\hline AM & $4.903,82$ & 258,09 & $4.898,92$ & 4,90 & - & \\
\hline Oxígeno & - & 0,05 & - & - & - & \\
\hline Quinona & 50,32 & 50,32 & 50,31 & 0,01 & - & \\
\hline Nitrógeno & - & 0,11 & - & - & - & \\
\hline Total & $12.139,56$ & $2.463,41$ & $5.027,14$ & $7.112,42$ & 800 & \\
\hline
\end{tabular}

Fuente: elaboración propia. 
De acuerdo con los resultados mostrados en la tabla 4, la corriente de salida del reactor (corriente número 10 en la figura 2) contiene alrededor de 6,09\% de AM, siendo los principales compuestos encontrados en esta corriente el nitrógeno y el oxígeno ( 74,88 y 10,37 $\%$, respectivamente). Cerca de $98,21 \%$ del benceno alimentado al reactor se convirtió en productos, quedando $\sin$ reaccionar $79,40 \mathrm{~kg} / \mathrm{h}$ de este compuesto, el cual compone cerca de $0,09 \%$ de la corriente de salida del reactor.

En el absorbedor se remueven los compuestos oxígeno, nitrógeno y dióxido de carbono por la corriente del tope de este equipo ( $\left.n .^{\circ} 16\right)$ con remoción de $99,99 \%$ para cada uno, por lo que se considera que la remoción de los gases incondensables es eficiente. En ese sentido, resulta válido precisar que esta corriente del tope está compuesta por 6,57 \% de dióxido de carbono, emitiéndose a la atmósfera un total de 5.160,76 $\mathrm{kg} / \mathrm{h}$ de este compuesto gaseoso. Como se planteó anteriormente, se recomienda efectuar estudios de ingeniería para realizar operaciones de separación, purificación y recobrado del $\mathrm{CO}_{2}$ emitido en esta corriente, con el fin de que sea comercializado como subproducto de valor económico agregado y además mitigar la emanación de gases de efecto invernadero a la atmósfera, así como incrementar la rentabilidad económica global del proceso productivo.

El agua, el benceno y la quinona se remueven en el absorbedor con las siguientes relaciones: 30,13 \%, 76,21 $\%$ y $1,24 \%$, respectivamente, mientras que el AM es recuperado en un $99,37 \%$ por el solvente $D F$, es decir, la pérdida de $\mathrm{AM}$ en este equipo es de solamente $0,63 \%(32,82 \mathrm{~kg} / \mathrm{h})$. Por último, solo $0,03 \%$ del DF alimentado al absorbedor se pierde por la corriente del tope, por lo que se puede decir que este equipo opera adecuadamente.

En el separador de fases, se remueve por la corriente del tope $\left(n .^{\circ} 20\right)$ la totalidad del benceno, $\mathrm{CO}_{2}, \mathrm{~N}_{2}$ y $\mathrm{O}_{2}$, el $95 \%$ del agua - que es el compuesto que se desea remover con mayor interés en esta etapa- y el $50 \%$ de la quinona. Por otro lado, se pierden $790,51 \mathrm{~kg} / \mathrm{h}$ de DF por la corriente del tope de este equipo, por lo tanto será necesario adicionar $800 \mathrm{~kg} / \mathrm{h}$ de DF fresco en el absorbedor para compensar esta pérdida. El AM se remueve en un $5 \%$, lo que significa que el $95 \%$ del AM alimentado al separador de fases $(4.903,82 \mathrm{~kg} / \mathrm{h})$ se recupera por la corriente del fondo de este equipo y es enviado hacia la columna de destilación para su purificación final. Por ello, el separador de fases funciona eficientemente, sirviendo de etapa de purificación intermedia del proceso productivo.

Por último, la columna de destilación recupera los compuestos AM y DF en un 99,89 y $99,86 \%$, respectivamente, obteniéndose por la corriente del fondo $\left(n .{ }^{\circ}\right.$ 13) el solvente DF con una pureza de $99,93 \%$, mientras que el producto deseado (AM) se recupera por la corriente del tope de este equipo $\left(n .^{\circ} 17\right)$ con una pureza de $97,45 \%$, siendo el agua $(1,41 \%)$, la quinona ( $1 \%)$ y el DF $(0,14 \%)$ las principales impurezas encontradas.

\section{Parámetros de diseño de los equipos}

La tabla 5 expone los principales parámetros de diseño y de operación determinados para cada uno de los equipos utilizados en el proceso, los cuales fueron calculados por el simulador ChemCAD ${ }^{\circledR}$.

Tabla 5. Parámetros de diseño y operación de cada uno de los equipos empleados en el proceso de producción

\begin{tabular}{|lll|}
\hline Bomba centrífuga & & \\
\hline Parámetro & $\mathrm{UM}$ & Valor \\
\hline Potencia calculada & $\mathrm{kW}$ & 0,35 \\
\hline Carga & $\mathrm{m}$ & 23,49 \\
\hline Caudal volumétrico & $\mathrm{m}^{3} / \mathrm{h}$ & 5,11 \\
\hline
\end{tabular}




\begin{tabular}{|lcc|}
\hline & Compresor & \\
\hline Parámetro & UM & Valor \\
\hline $\mathrm{Cp} / \mathrm{Cv}$ ideal & & 1,39 \\
\hline $\mathrm{Cp} / \mathrm{Cv}$ & & 1,40 \\
\hline Potencia teórica & $\mathrm{kW}$ & 25,36 \\
\hline Potencia real & $\mathrm{kW}$ & 31,71 \\
\hline
\end{tabular}

\begin{tabular}{|lccc|}
\hline \multicolumn{4}{|c|}{ Intercambiadores de calor } \\
\hline Parámetro & UM & Precalentador & Enfriador \\
\hline Carga de calor calculada & MJ/h & $2.347,77$ & $35.504,10$ \\
\hline Media Logarítmica de la Diferencia de Temperatura (MLDT) & oC & 46,20 & 125,06 \\
\hline Caudal de servicio auxiliar & $\mathrm{kg} / \mathrm{h}$ & 1094,92 & 21239,36 \\
\hline
\end{tabular}

\begin{tabular}{|lccc|}
\hline \multicolumn{3}{|c|}{ Calentador quemador } \\
\hline Parámetro & UM & Valor \\
\hline Calor absorbido & & $\mathrm{MJ} / \mathrm{h}$ & $19.634,50$ \\
\hline
\end{tabular}

\begin{tabular}{|lccc|}
\hline \multicolumn{3}{|c|}{ Reactor } & \\
\hline Parámetro & UM & Valor \\
\hline Calor total de reacción & MJ/h & $-8.145,28$ \\
\hline
\end{tabular}

\begin{tabular}{|lccc|}
\hline \multicolumn{3}{|c|}{ Absorbedor } & \\
\hline Parámetro & UM & Valor \\
\hline Número total de etapas & - & 14 \\
\hline
\end{tabular}

\begin{tabular}{|lcc|}
\hline \multicolumn{2}{|c|}{ Columna de destilación } & \\
\hline Parámetro & UM & Valor \\
\hline Carga de calor en el condensador & $\mathrm{MJ} / \mathrm{h}$ & $-4.622,24$ \\
\hline Demanda de agua de enfriamiento en el condensador & $\mathrm{kg} / \mathrm{h}$ & 7.245 \\
\hline Carga de calor en el rehervidor & $\mathrm{MJ} / \mathrm{h}$ & $10.823,60$ \\
\hline Demanda de vapor de agua en el rehervidor & $\mathrm{kg} / \mathrm{h}$ & 11.578 \\
\hline Etapas & - & 15 \\
\hline Etapa de alimentación & - & 11 \\
\hline Relación de reflujo calculada & - & 1,35 \\
\hline
\end{tabular}

Fuente: elaboración propia. 
La tabla 5 muestra que la bomba a utilizar para bombear el benceno deberá tener una potencia de 0,35 kW y una carga de 23,49 m, así como un caudal de diseño de $5,11 \mathrm{~m}^{3} / \mathrm{h}$.

La potencia real que deberá poseer el compresor es de 31,71 kW. En los intercambiadores de calor, el enfriador presenta un valor de carga de calor 15 veces superior al del precalentador, lo cual se debe a que en el enfriador se lleva a cabo una importante reducción de la temperatura de la mezcla gaseosa reaccionante de salida del reactor desde 380 ㅇ C hasta 54 ㅇ C (diferencia de 326 ㄷ) , que origina una gran liberación de calor por parte del material que está siendo enfriado. Además, en el enfriador se obtiene una media logarítmica de la diferencia de temperatura (MLDT) 2,7 veces superior en comparación con aquella calculada para el precalentador, lo cual se debe a los amplios límites de temperatura manejados en este intercambiador. Por otro lado, se necesitan $21.239,36 \mathrm{~kg} / \mathrm{h}$ de agua de enfriamiento en el enfriador para hacer frente a la carga de calor requerida, mientras que resulta necesario utilizar 1.094,92 kg/h de vapor de agua por el precalentador para calentar la corriente de bence-

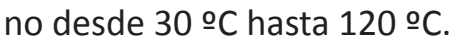

En el calentador quemador se absorben 19.634,50 $\mathrm{MJ} / \mathrm{h}$ de calor por parte de la mezcla gaseosa benceno/aire con el fin de incrementar su temperatura desde $169 \stackrel{\circ}{\circ}$ hasta $380 \stackrel{\circ}{ }$.

El calor de reacción total calculado para las cuatro reacciones químicas que ocurren en el reactor es de - 8.145,28 MJ/h, lo cual corrobora la afirmación de que las reacciones son exotérmicas.

El absorbedor necesita 14 etapas para efectuar su operación, mientras que la columna de destilación necesita 15 etapas, siendo la etapa de alimentación la número 11. La remoción de calor en el condensador es de $-4.622,24 \mathrm{MJ} / \mathrm{h}$, mientras que la carga de calor en el rehervidor es de 10.823,6 MJ/h. Por último, se necesita una relación de reflujo calculada para esta columna de 1,35.

\section{Curvas de calor}

La figura 3 muestra las curvas de calor obtenidas mediante el simulador ChemCAD ${ }^{\circledR}$ para cada uno de los intercambiadores de calor empleados en el proceso de producción.

Figura 3. Curvas de calor obtenidas para los dos intercambiadores de calor empleados en el proceso: precalentador (izquierda) y enfriador (derecha)
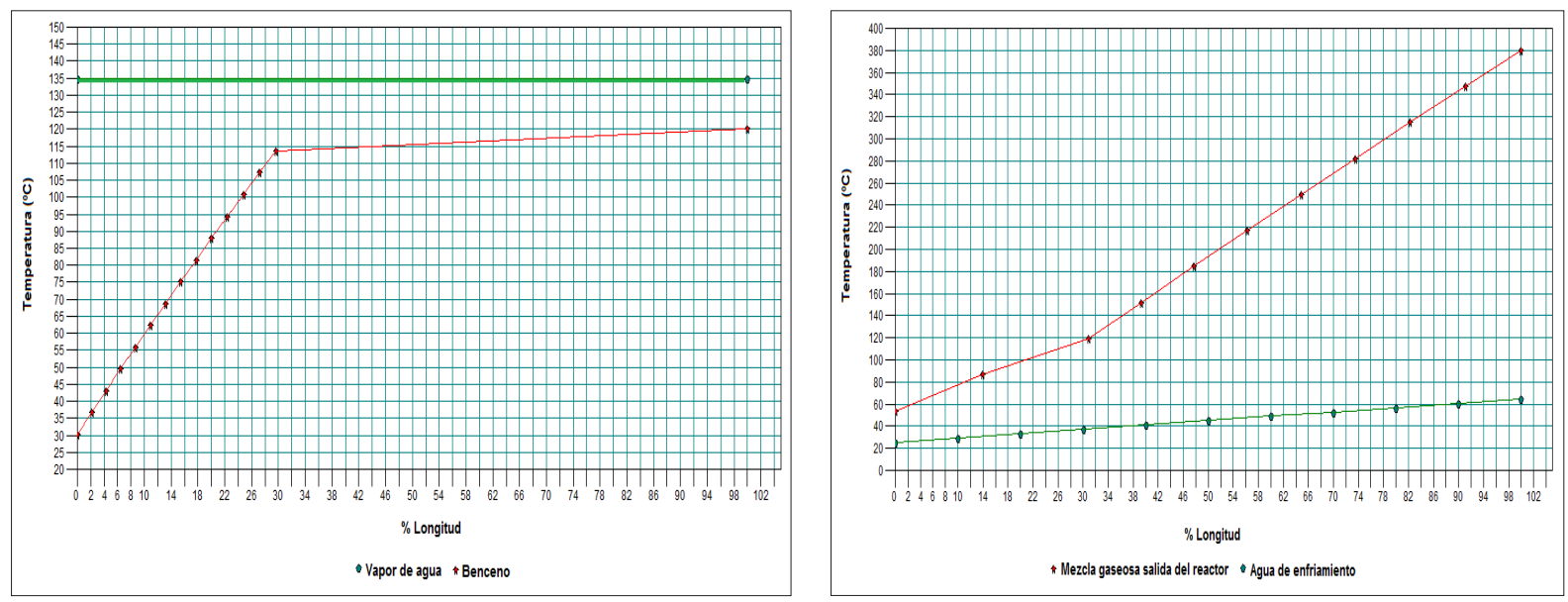

Fuente: elaboración propia. 
Las curvas de calor obtenidas para el precalentador indican que el vapor de agua experimenta un cambio de fase a la temperatura de alimentación (135 ㄷ), lo que significa que este servicio entra en condiciones saturadas a dicho intercambiador de calor. El benceno se calienta sin cambio de fase hasta los 115 ㅇ $\mathrm{y}$ una longitud del intercambiador del $30 \%$, cambiando de fase y continuando el calentamiento según la tendencia lineal creciente que se observa en su curva de calor. A partir de este punto ocurre la vaporización de este compuesto hasta alcanzar la temperatura de salida deseada $(120 \stackrel{\circ}{\circ})$, manifestándose mediante el cambio de patrón (punto de giro) obtenido para esta curva.

Finalmente, considerando las curvas de calor obtenidas para el enfriador, se deduce que la mezcla reaccionante tolera enfriamiento sin ocurrir cambio de fase hasta una temperatura de 120 ㅇ C y una longitud del intercambiador del $31 \%$, a partir de la cual ocurre cambio de fase de algunos de sus componentes hasta alcanzar la temperatura de salida deseada (54 으), manifestado por medio del cambio de patrón observado en la curva obtenida. La curva de calor del agua de enfriamiento demuestra por su parte que solo absorbe calor sensible.

Figura 4. Resultados del estudio de sensibilidad realizado

a)

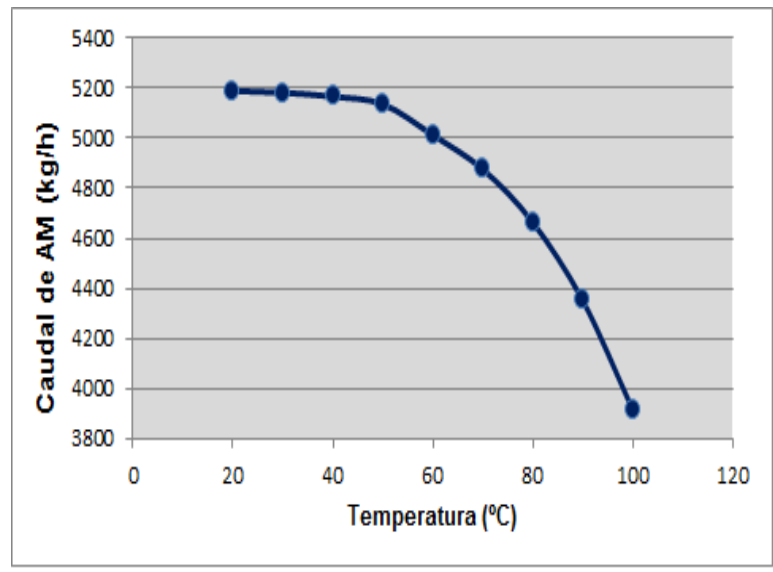

\section{Estudios de sensibilidad}

La figura 4 muestra los resultados obtenidos con respecto al estudio de sensibilidad efectuado. Según los resultados obtenidos en el estudio de sensibilidad n. ${ }^{\circ} 1$ (figura 4a), a medida que aumenta la temperatura de operación del absorbedor disminuye la cantidad a obtener de AM por la corriente del fondo, es decir, existe una mayor evaporación de este compuesto por la corriente del tope. Lo anterior se deduce a partir de la tendencia curvada decreciente observada, siendo evidente a partir de los 50 ㅇ. . Así, un incremento de la temperatura de operación desde 40 hasta 50 oC producirá pérdidas de $A M$ de $30,93 \mathrm{~kg} / \mathrm{h}$, un incremento de $50 \stackrel{\circ}{\circ}$ hasta $60 \cong$ C ocasionará que se pierdan $123,12 \mathrm{~kg} / \mathrm{h}$ de $\mathrm{AM}$ y un aumento de 90 으 hasta 100 -C causará pérdidas de AM de $436,05 \mathrm{~kg} / \mathrm{h}$, es decir, la tendencia es ciertamente creciente a medida que aumenta la temperatura de operación del absorbedor.

En cuanto al estudio de sensibilidad $n .^{\circ} 2$ (figura $4 b$ ), se deduce que un incremento de la presión de operación del absorbedor favorece una mayor obtención de AM por la corriente del fondo de este equipo. Según los resultados obtenidos, un incremento de la presión desde 1 bar hasta 5 bar ocasionará que se recuperen 229,26 $\mathrm{kg} / \mathrm{h}$ de $\mathrm{AM}$, mientras que un aumento desde 1 bar hasta 10 bar producirá que se recobren $242,91 \mathrm{~kg} / \mathrm{h}$.

a) Temperatura de operación del absorbedor versus caudal de AM a obtener por la corriente de fondo de este equipo; b) presión de operación del absorbedor versus caudal de $\mathrm{AM}$ a obtener por la corriente de fondo de este equipo.

Fuente: elaboración propia. 
Tomando en cuenta que el costo unitario del AM en el mercado internacional se encuentra en el orden de USD 2,68/kg (ICIS, 2021), el incremento de la temperatura de operación en el absorbedor des-

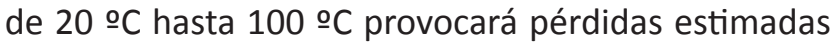
de USD 3.412,02 debido a la evaporación (salida) de AM por la corriente del tope de este equipo. Por su parte, el incremento de la presión de operación en el absorbedor desde 1 bar hasta 5 bar posibilitará que se generen ganancias económicas adicionales de USD 614,42 a causa del incremento de la cantidad de AM obtenido por la corriente del fondo.

De esta manera, se recomienda operar el absorbedor a una temperatura cercana a 20 으 y una presión de 5 bar, con el fin de maximizar las cantidades a obtener de AM por la corriente del fondo de este equipo y, con ello, las ganancias económicas.

\section{CONCLUSIONES}

El empleo del simulador ChemCAD ${ }^{\circledR}$ permitió simular de forma eficiente el proceso de producción de anhídrido maleico a partir de la oxidación catalítica del benceno.

El precalentador necesita 1.094,92 kg/h de vapor de agua y el enfriador $21.239,36 \mathrm{~kg} / \mathrm{h}$ de agua de enfriamiento para llevar a cabo sus operaciones térmicas.

Se obtienen $5.027,14 \mathrm{~kg} / \mathrm{h}$ por la corriente del tope de la columna de destilación, conteniendo AM con una pureza de $97,45 \%$, siendo el agua la principal impureza encontrada con un $1,41 \%$ del total, mientras que el AM recobrado de este compuesto en este equipo alcanzó 99,89 \%.

El absorbedor debe operar a una temperatura cerca-

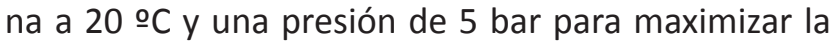
cantidad de AM a obtener por la corriente del fondo $y$, con ello, las ganancias económicas.

\section{REFERENCIAS}

Almena, A., \& Martín, M. (2016). Technoeconomic analysis of the production of epichlorohydrin from glycerol. Industrial \& Engineering Chemistry Research, 55, 3226-3238. https://doi. org/10.1021/acs.iecr.5b02555

Cao, E. (2010). Heat transfer in process engineering. McGraw-Hill.

Chilev, C., \& Simeonov, E. (2014). Simulation of biodiesel production by transesterification of vegetable oils. Journal of Chemical Technology and Metallurgy, 49(5), 479-486.

Eliasson, J. (2010). Design of a plant for manufacturing acetaldehyde. Lund University.

Fernández, J. R., Vega, A., \& Díez, F. V. (2010). Partial oxidation of $n$-butane to maleic anhydride over VPO in a simulated circulating fluidized bed reactor. Applied Catalysis A: General, 376(1-2), 76-82. https://doi.org/10.1016/j. apcata.2009.12.006

Greeshma, N., Shah, B. H., \& Patel, N. M. (2014). Simulation study of reactive distillation for monochloroacetic acid using ChemCAD. International Journal of Futuristic Trends in Engineering and Technology, 1(2), 1-11.

Independent Commodity Intelligence Services [ICIS] (2021). Global maleic anhydride market outlook. https://www.icis.com/explore/commodities/ chemicals/maleic-anhydride/

Kirk, R. E., Othmer, D. F., Grayson, M., \& Eckroth, D. (eds.) (2004). Kirk-Othmer encyclopedia of chemical technology (cuarta ed., vols. 4, 15, 20). John Wiley \& Sons.

Nosakhare, F., Yakubu, A. M., \& Onajite, R. O. (2017). Design of a 40,000 tonnes capacity maleic anhydride production process using benzene as feedstock (tesis de pregrado, Universidad de Benín). Universidad de Benín. 
Pérez, A., Pérez, E. J., \& Segura, R. M. (2017). Simulation of the styrene production process via catalytic dehydrogenation of ethylbenzene using ChemCAD process simulator. Tecnura, 21(53), 1531. https://doi.org/10.14483/22487638.11499

Pérez, A., Pérez, E. J., \& Segura, R. M. (2019). Simulation of the acrylic acid production process through catalytic oxidation of gaseous propylene using ChemCAD $^{\circ}$ simulator. Ingeniare, 27(1), 142-150. https://doi.org/10.4067/ S0718-33052019000100142

Pugsley, T. S., Patience, G. S., Berruti, F., \& Chaouki, J. (1992). Modeling the catalytic oxidation of n-butane to maleic anhydride in a circulating fluidized bed reactor. Ind Eng Chem Res., 31, 2652-2660. https://doi.org/10.1021/ie00012a005

Rabelo, Y. (2016). Simulación del proceso de producción de una fábrica de conservas de frutas y vegetales (tesis de maestría, Instituto Superior Politécnico José Antonio Echeverría). Instituto Superior Politécnico José Antonio Echeverría.

Speight, J. (2002). Chemical and process design handbook. McGraw-Hill.

Tandioy, O. M., Gil, I. D., \& Sanchez, O. F. (2009). Modeling of maleic anhydride production from a mixture of $\mathrm{n}$-butane and butenes in fluidized bed reactor. Latin American Applied Research, 39, 19-26.
Tubular Exchanger Manufacturers Association [TEMA]. (1999). Standards of the Tubular Exchanger Manufacturers Association (octava ed.). TEMA.

Turton, R., Shaeiwitz, J. A., Bhattacharyya, D., \& Whiting, W. B. (2018). Analysis, synthesis, and design of chemical processes (quinta ed.). Prentice Hall.

Uraz, C., \& Atalay, S. (2007). Oxidation of benzene to maleic anhydride in a fluidized bed reactor. Chem Eng Technol., 30(12), 1708-1715. https://doi. org/10.1002/ceat.200700249

Uraz, C., \& Atalay, S. (2012). Comparison between the performances of a fluidized-bed reactor and a fixed-bed reactor for the oxidation of benzene to maleic anhydride. Turkish J Eng Env Sci., 36, 59-71.

Wyczesany, A. (2017). Simulation of n-propanol dehydration process via heterogeneous azeotropic distillation using the NRTL Equation. Chemical and Process Engineering, 38(1), 163175. https://doi.org/10.1515/cpe-2017-0013 